\title{
Heavy Minerals in the
}

\section{Pleistocene Terraces of South Carolina and Georgia}

\section{Trace Elements Memorandum Report 23}

UNITED STATES DEPARTMENT OF THE INTERIOR GEOLOGICAL SURVEY 


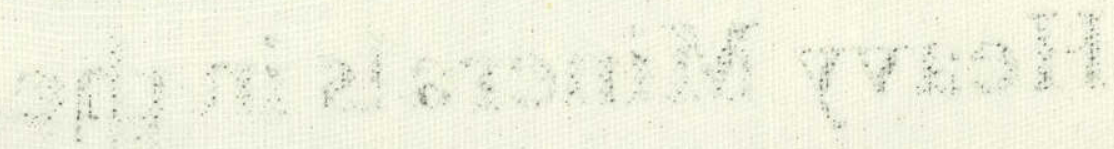

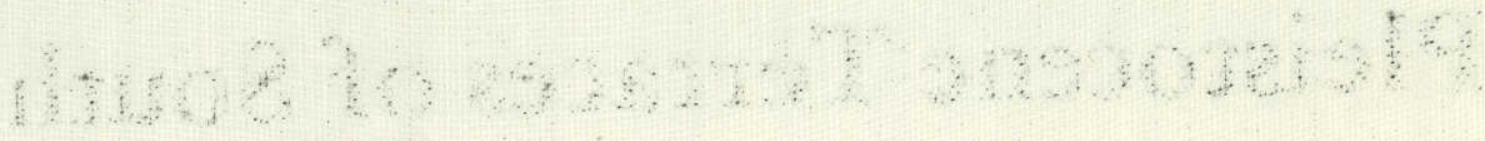

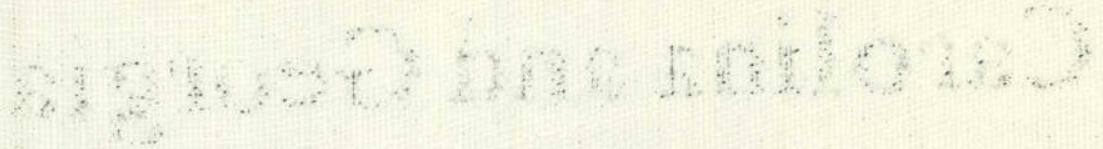


UNCLASSIF IED

This document consists of 27 pages, Series A

CATEGORY VIII (Deposits East of Rocky Mountains)

UNITED STATES DEPARTMENT OF THE INTERICR

GEOLOGICAL SURVEY

HEAVY MDISRALS IN THE PLEISTOCENE TERRACE

DEPOSITS OF SOUTH GAPOITDIA UPORGIA

By

John B. Mertie, Jr.

June 1951

Trace Element Memorandum Report 23 
USGS - TEM Report 23

The distribution (Series A) of this report is as follows:

3 copies .... AEC, Washington (J. C. Johnson)

9 copies . . . AEC, New York (P. I. Merritt)

$I$ copy ..... $A E C$, Denver, Colo. (C. C. Towle, Jr. )

1 copy ..... AEC, Spokane, Wash. (E. E. Thurlow)

1 copy ..... AEC, Grand Junction, Colo. (T. W. Oster)

1 copy ..... Columbia Univ., New York (P. F. Kerr)

I copy ..... Univ. Minnesota, Minneapolis (J. W. Gruner)

9 copies . . U USGS, Washington (Minergl Deposits Branch)

1 copy ..... USGS, Washington (Geochemistry and Petrology Branch)

1 copy ..... USGS, Washington (Geophysics Branch)

1 copy ..... USGS, Washington (Fuels Branch)

1 copy ..... USGS, Washington (Alaskan Branch)

1 copy ..... USGS, Washington (V. E. McKelvey)

1 copy ..... USGS, Denver, Colo. (L. R. Page)

1 copy ..... USGS, Grand Junction, Colo. (R. P. Fischer)

1 copy ..... USGS, Spokane, Wash. (A. E. Weissenborn)

1. copy ..... USGS, Plant City, Fla. (J. B. Cathcart)

4 copies .... USGS, Washington (TEPCO)

(Including master copy) 
CONTENTS

Prge

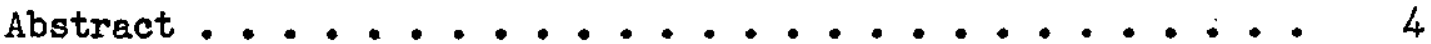

Introduction ...................... 5

General geology. . . . . . . . . . . . . 5

Samples. ...................... 8

Southeastern South Carolina .......... 8

Southeastern Georgia. ............. 21

Conclustons. ................... 27

\section{IILUSTRATIONS}

Figure 1. Heavy mineral samples, southeastern South Carolina. 9

2. Heavy mineral samples, southeastern feorgia. . . 22

TABLES

Table 1. Localities and descriptions of samples from southeastern South Carolina..... . . 10-17

2. Heavy mineral content of samples from Pleistocene terrace deposits and Eocene formations in southeastern South Carolina................18-19

3. Localities and descriptions of samples from southeastern Georgia. . . . . . . 23-25

4. Heavy mineral content of samples from Pleistocene terrace deposits in southeastern Georgia ..... 26 
HEAVI IIINFRALS IN THE PLEISTCCENE TERRACE

DEPOSITS OF SOUTH CAROLINA AND GEORGIA

By

John B. liertie, Jr.

ABSTRACT

The Pleistocene terrace deposits of southeastern South Carolina and southeastern Georgia contain minor amounts of heavy minerals. A prel minary examination, which was made of these terraces in October 1948 , indicates that if economic concentration of heavy minerals exist in the Pleistocene terrace deposits of South Carolina and Georgia, they most likely occur in the Wicomico, Okefenokee, and Sunderland terraces and to the east and southeast of Trail Ridge, Georgia. Exploration for these terrace deposits could be done by deep drilling with casing, although shallow exploretion down to the vater table could be done by drilling without casing or by trenching.

The mean percentage of heavy minerals in samples from terrace deposits in southeastern Georgia is 0.37 , whereas the mean percentage of heavy minerals in samples from terrace deposits in southeastern South Carolina is 0.15 . Sands containing relatively large amounts of heavy minerals contain small percentages of monazite; sands containing relatively small amounts of heavy minerals contain large percentages of monazite. 
INTRODUCTION

Interest was shown, by the Atomic Energy Commission, in the possibility of the existence of large, even though low-grade, deposits of heavy minerals in the Pleistocene terraces of North Carolina, South Carolina, and Georgia. Particular interest was shown in the Pleistocene terraces that are adjacent to streams that drain the western monazite belt of North Carolina and South Carolina. Accordingly, a preliminary examination of the southeastern Coastal Plain was made in October 1948, during which 56 samples were taken of the Pleistocene terrace deposits and concentrated by panning in the field. The mineralogic work on these samples was done by the Trace Elements laboratory in Washington, D. C.

GENERAL GEOLOGY

The Broad and Saluda Rivers, which drain most of the western monazite belt of North and South Carolina, join to form the Congaree River; and the Congaree joins the Waterlee to form the Santee River, which flows 100 miles to the Atlantic Ocean. This drainage system has persisted with minor modifications throughout Pleistocene and Recent times. All of the Pleistocene terraces that were directly controlled by Atlantic base-levels occur within or close to the valley of the Santee River; therefore a preliminary examination was made of the terraces of this part of southeastern South Carolina. Additional work was done on the Pleistocene terraces of southeastern Georgia. 
Eight Pleistocene terraces have been described by Cooke_,

\begin{abstract}
$\downarrow$ Cooke, C. W., Seven coastal terraces in the southeastern states: Washington Acad. Sci. Jour., vol. 2l, no. 21, pp. 503-513, 1931.

Geology of the Coastal Plain of Georgia: U. S. Geol. Survey Bull. 941, 1944.
\end{abstract}

Stephenson $/$, and other geologists who have worked in the Atlantic

\begin{abstract}
J Stephenson, I. W., Major features in the geology of the Atlantic and Gulf Coastal Plain: Washington Acad. Sci., Jour., rol. 16, no. 17, pp. 460-480, 1926.
\end{abstract}

Coastal Plain. Terraces, from the highest to the lowest, are: the Brandywine, Coharip, Sunderland, Orefenokep, Wi comich, Ponholowar, Talbot, and Pamlico. The maximum altitudes of these terraces are respectively $300,215,170,140,100,70,42$, and 25 feet. The upper limit of each of these formations has been determined in some measure by scarps and other physiographic features; but where such significant data are lacking, it has been defined by hypsometry. Neither lithologic nor paleontologic definitions of these formations have been presented.

Most of the terrace deposits in and near the Santee Valley contain differing amounts of silt and clay. The master streams that debouched during the Pleistocene epoch onto the swampy forelands bordering the ocean were muddy, though probably not to the degree that they now are. The resulting deposits were therefore originally 
clayey, and most have remained so. Some of these deposits were reworked by the ocean in the course of numerous advances and regressions of the strand-line, but most of them are not trwe marine sands

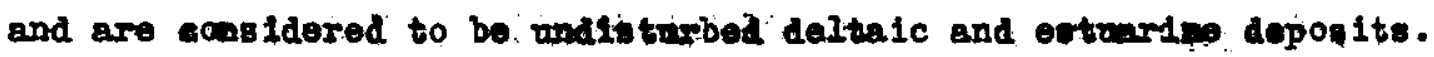
Littoral reconcentration of the heavy minerals in these deposits did not occur.

Conditions of similar Pleistocene sedimentation probably prevailed throughout North Carolina and South Carolina, and most of Georgia, where large rivers heading in the Pledmont belt reach the ocean.

South of the Altamaha River in southeastern Georgia, many of the terrace deposits were reworked by oceanic waves and currents, and therefore could be classified as marine deposits. Former shorelines have been recognized in the Wicomico, Okefenokee, and Sunderland formations along the eastern flank of Trail Ridge. Prospecting of these formations in this area, and to the east and southeast, is more likely to be rewarding than searching for concentrations of heavy minerals in the area to the northeast. A large deposit of heavy minerals was discovered in Florida, near the south end of Trail Ridge, and has been mined on a large scale by the Humphrey's Gold Corporation since 1949. 


\section{SAMPLES}

\section{Southeastern South Carolina}

Thirty-nine samples of the Pleistocene formations in and near the Santee Valley of South Garolina, were panneduffignil). Fatw other samples from the same area were taken of the McBean and Black Mingo formations of middle and lower Eocene age, respectiveIy. The localities, altitudes, terrace assignments, lithology, and content of heavy minerals for all these samples are given in the tables 1 and 2 .

Owing to the interest of the Atomic Energy Comission in the discovery of deposits of heavy minerals containing significant amounts of monazite, more field work was done in the Santee River valley of South Carolina than in southeastern Georgia. Though the headwater tributaries of this river drain much of the western Carolina monazite belt, the results of the examination indicate that heavy minerals make up an average of only 0.15 percent of the terrace sands and that only 2.7 percent of the heavy minerals consist of monazite. Monazite then makes up about 0.004 percent of the terrace deposits, a grade no higher than that of the monazitebearing granite of Shelby, North Carolina.

On the basis of the few samples taken during this examination, the Eocene formations of the area appear to contain twice as high a percentage of monazite as the terraces, but the total content of heavy minerals appears to be even smaller than that of the terraces. 


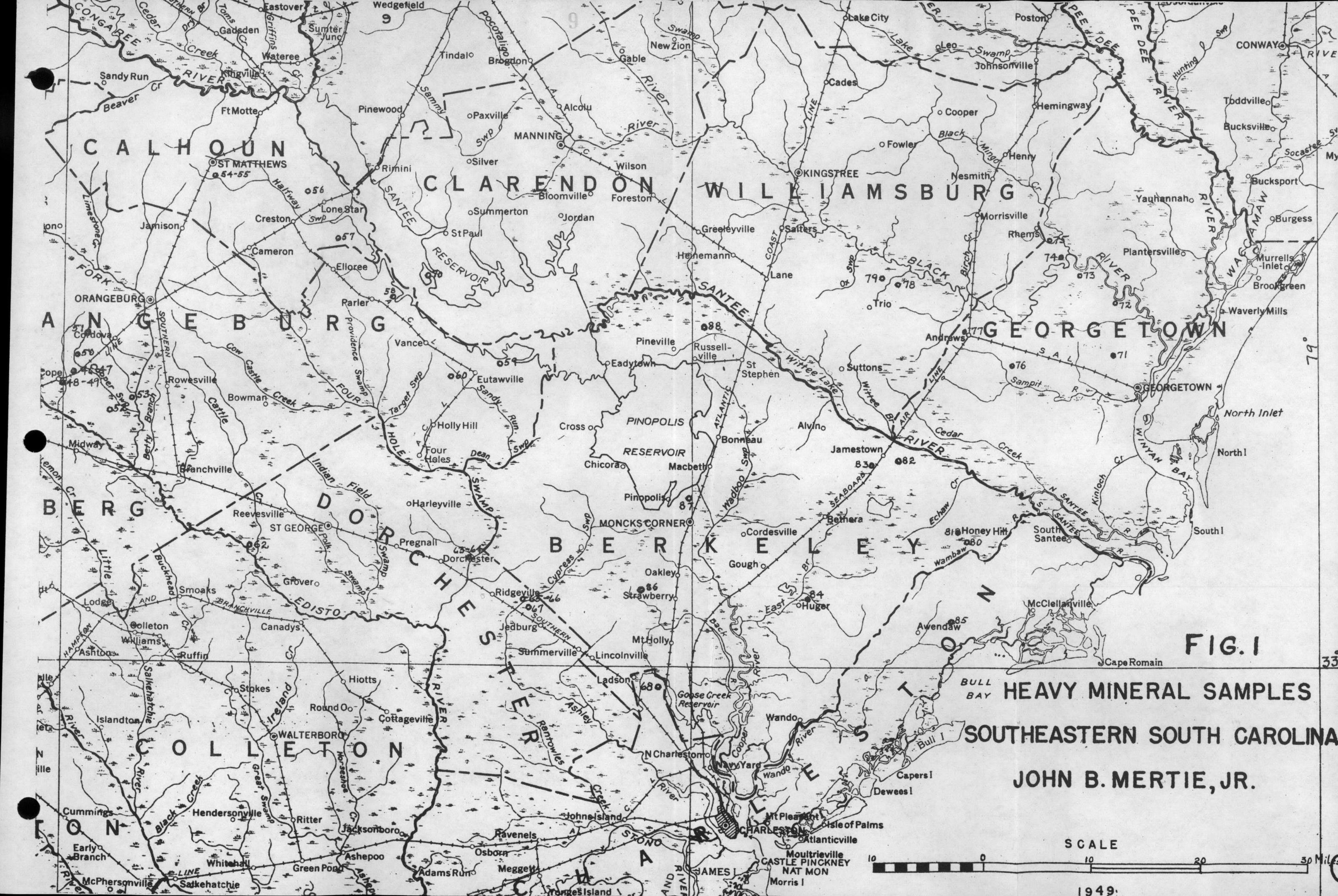


Table 1.--Localities and descriptions of samples from southeasterm South Carolina.

\section{Sample No. Location}

$48 \mathrm{Mt} 46$ $1 / 4$ mile northeast of
Snake Swamp on State Snake Swamp on State County.

48 Mit 47 $"$

$n$

$48 \mathrm{Mt} 48$ Quarry $1 / 8$ mile south- Coharie terrace west of Snake Swamp on and formation. State road 33, Orangeburg County.

$48 \mathrm{Mt} 49$ " "1 $n$

$48 \mathrm{Mt} 50$

Sideroad running north- Brandywine terrace west from State road 33 and formation. and 0.2 mile southeast of Cooper Swamp. Also 8.9 miles $S 54^{\circ} W$ from center of Orangeburg, Orangeburg County.

$48 \mathrm{Mt} 51$ On Bennecker Bridge Brandywine terrace road, 7.1 miles $S 62^{\circ} \mathrm{W}$ and formation. from center of Orangeburg, Orangeburg County.

Coharie terrace
and formation.

Altitude tion sampled (pounds) Formation (above sea level) Description fine-grained, clayey, sandy

$185^{\circ}$ material.

Clayey, reddish-brown Iimonitic Upper 4 feet of material. section. 25

$170^{\prime}$

Mottled light tan to brown to red, quartzose grit with pebbles up to $1 / 2^{\prime \prime}$ in size.

6 feet of

6 exposed feet.
Sandy material. up to $1 / 2$ inch in diameter. 


\begin{tabular}{|c|c|c|c|c|c|c|c|}
\hline Sample No. & Location & Formation & $\begin{array}{l}\text { titude } \\
\text { sea level) }\end{array}$ & Description & $\begin{array}{l}\text { Part of forma- } \\
\text { tion sampled }\end{array}$ & $\begin{array}{l}\text { Sample } \\
\text { weight } \\
\text { (pounds) } \\
\end{array}$ & $\begin{array}{l}\text { Heavy } \\
\text { mineral } \\
\text { weight } \\
\text { (grams) }\end{array}$ \\
\hline $48 \mathrm{Mt} 52$ & $\begin{array}{l}\text { Southside of State road } \\
36 \text { about } 0.45 \text { nile west } \\
\text { of Cooper Swasip and } 10.0 \\
\text { miles S } 20^{\circ} \text { W. from } \\
\text { center of Orangeburg, } \\
\text { Orangeburg County. }\end{array}$ & $\begin{array}{l}\text { sunderland terrace } \\
\text { and cormation. }\end{array}$ & $155^{\prime}$ & $\begin{array}{l}\text { Slightly clayey, quartzose } \\
\text { grit, quartz grains from very } \\
\text { small size up to } 3 / 8 \text { inch in } \\
\text { diameter. }\end{array}$ & 3 feet & 25 & 8.0 \\
\hline 48 Mt 53 & $\begin{array}{l}\text { North side of State road } \\
36 \text {, about } 0,1 \text { mile west } \\
\text { of North Fork of Kdis to } \\
\text { River; and } 9.65 \text { mfles S } \\
11 \frac{10}{2} \text { W from center of Oras } \\
\text { burg, Orangeburg County: }\end{array}$ & $\begin{array}{l}\text { Sunderland terrace } \\
\text { ange- }\end{array}$ & $150^{\prime}$ & $\begin{array}{l}\text { Reddish-brown to yellowish, } \\
\text { clayey sand, }\end{array}$ & $\begin{array}{l}\text { Lower S-feet } \\
\text { ol } 9 \text {-joot } \\
\text { section } \\
\end{array}$ & 25 & 3.2 \\
\hline 48 lit 54 & $\begin{array}{l}\text { West side of U. S. road } \\
21 \text {, about } 1 \frac{1}{4} \text { miles south } \\
\text { of St. Matthews, Calhoun } \\
\text { County. }\end{array}$ & $\begin{array}{l}\text { McBean (middle } \\
\text { Eocene) formation }\end{array}$ & $?$ & $\begin{array}{l}\text { Coarse-grained, quartzose } \\
\text { grit containing marine fossils. }\end{array}$ & $\begin{array}{l}\text { Upper } 5 \text { feet } \\
\text { of } 15 \text { feet }\end{array}$ & 25 & 2.2 \\
\hline 48 Mt 55 & $"$ & " & ? & $"$ & $\begin{array}{l}\text { Lower } 5 \text { feet } \\
\text { of } 15 \text { feet }\end{array}$ & 25 & 3.7 \\
\hline $48 \mathrm{Mt} 56$ & $\begin{array}{l}\text { Southwest side of State } \\
\text { road } 47,1.3 \text { miles west- } \\
\text { northwest of junction of } \\
\text { State roads } 47 \text { and } 33 \text {, } \\
\text { Calhoun County. }\end{array}$ & $\begin{array}{l}\text { Dune behind Coharie } \\
\text { terrace and fornatic }\end{array}$ & $\begin{array}{l}230^{\prime} \\
\text { on. }\end{array}$ & $\begin{array}{l}\text { Unconsolidated yellow sand } \\
\text { with darker irregular-shaped } \\
\text { masses. }\end{array}$ & $\begin{array}{l}\text { Lower } 5 \text { feet } \\
\text { oi } 8 \text {-foot bluff }\end{array}$ & 25 & 35.3 \\
\hline 48 Mt 57 & $\begin{array}{l}\text { East side of State road } \\
47 \text {, about } 33 / 4 \text { miles } S \\
23^{\circ} \mathrm{E} \text { of Lone Star, } \\
\text { Calhoun County. }\end{array}$ & $\begin{array}{l}\text { Sunderland terrace } \\
\text { and formation, }\end{array}$ & $110^{\prime}$ & $\begin{array}{l}\text { Reddish-brown consolidated } \\
\text { sandstone, about } 2 / 3 \text { quartz } \\
\text { grains and } 1 / 3 \text { clay. }\end{array}$ & $\begin{array}{l}4 \text {-foot } \\
\text { section }\end{array}$ & 25 & 8.8 \\
\hline
\end{tabular}


Table 1.--(Continued)

\begin{tabular}{|c|c|c|c|c|c|c|c|}
\hline Sample No. & Location & $\begin{array}{r}\mathrm{Al} \\
\text { (above } \\
\end{array}$ & $\begin{array}{l}\text { titude } \\
\text { sea level) }\end{array}$ & Description & $\begin{array}{l}\text { Part of forma- } \\
\text { tion sampled }\end{array}$ & $\begin{array}{l}\text { Sample } \\
\text { weight } \\
\text { (pounds) } \\
\end{array}$ & $\begin{array}{l}\text { Heavy } \\
\text { mine ral } \\
\text { weight } \\
\text { (grams) } \\
\end{array}$ \\
\hline $48 \mathrm{Mt} 59$ & $\begin{array}{l}\text { State road } 45,1 / 2 \text { mile } \\
\text { west of Eutaw Spri gs, } \\
75 \text { yards east of junction } \\
\text { of State roads } 45 \text { and } 6 \text {, } \\
\text { Orangeburg vounty. }\end{array}$ & $\begin{array}{l}\text { Wickomico terrace } \\
\text { and formation. }\end{array}$ & $100^{\prime}$ & $\begin{array}{l}\text { Yellowish-brown uncon- } \\
\text { solidated sand. }\end{array}$ & 6-foot section & 25 & 5.6 \\
\hline $48 \mathrm{Mt} 60$ & $\begin{array}{l}\text { State road } 45 \text {, about } 2 \frac{1}{4} \\
\text { miles west of Eutawville, } \\
\text { Orangeburg County. }\end{array}$ & $\begin{array}{l}\text { Dune on Okefonokee } \\
\text { terrace and formation } \\
\end{array}$ & $140^{\prime}$ & Yellow sand. & 2 -foot section & 25 & 2.7 \\
\hline 48 Mt 62 & $\begin{array}{l}\text { Utsey Bluff on northeast } \\
\text { side of Edisto River, } \\
\text { O.7 mile southeast of } \\
\text { Beulah Church, and about } \\
5 \text { miles S } 45^{\circ} \text { w of Keeves- } \\
\text { ville, Dorchester Count,y. }\end{array}$ & $\begin{array}{l}\text { Wicomicoterrace } 87 \\
\text { and formation. }\end{array}$ & $-95^{\prime}$ & $\begin{array}{l}\text { Partially consolidated } \\
\text { gritty sandstone, that } \\
\text { disintegrates in water. }\end{array}$ & $\begin{array}{l}8 \text { feet, section } \\
\text { starting } 5 \text { feet } \\
\text { below surface. }\end{array}$ & 25 & 21.4 \\
\hline $48 \mathrm{Nt} 63$ & $\begin{array}{l}\text { North side of U. S. road } \\
78 \text {, about } 2 \frac{1}{2} \text { miles east, } \\
\text { and a little north of } \\
\text { Dorchester, and about } 30 \\
\text { yards west of Four Hole } \\
\text { Swarnp, Dorchester County. }\end{array}$ & $\begin{array}{l}\text { Penholoway terrace } \\
\text { and formation. }\end{array}$ & $70^{\prime}$ & $\begin{array}{l}\text { Cooper marl (Eocene) at } \\
\text { base overlain by } 3 \text { feet of } \\
\text { carbonaceous sand, over- } \\
\text { lain by } 2 \frac{1}{2} \text { feet of very } \\
\text { carbonaceous sand, over- } \\
\text { lain by } 15 \text { feet of yellow s, }\end{array}$ & $\begin{array}{l}\text { Lower } 12 \text { feet of } \\
15 \text {-foot section } \\
\text { of yellow sand }\end{array}$ & 25 & 15.5 \\
\hline
\end{tabular}


Table 1.-(Continued)

\begin{tabular}{|c|c|c|c|c|c|c|c|}
\hline Sample No. & Location & Formation & $\begin{array}{l}\text { titude } \\
\text { e sea level) }\end{array}$ & Description & $\begin{array}{l}\text { Part of forma- } \\
\text { tion sampled }\end{array}$ & $\begin{array}{l}\text { Sample } \\
\text { weight } \\
\text { (pounds) }\end{array}$ & $\begin{array}{l}\text { Heavy } \\
\text { mineral } \\
\text { weight } \\
\text { (grams) }\end{array}$ \\
\hline 48 lift 64 & $\begin{array}{l}\text { Same as preceeding lo- } \\
\text { cation ( } 48 \text { Mt 63) }\end{array}$ & $\begin{array}{l}\text { Penholoway terrace } \\
\text { and formation. }\end{array}$ & $70^{\prime}$ & $\begin{array}{l}\text { Same as preceeding } \\
\text { (48 Mt 63) }\end{array}$ & $\begin{array}{l}2 \frac{1}{2} \text { feet of } 2 \frac{1}{2} \\
\text { feet of very } \\
\text { carbonaceous sand }\end{array}$ & d & 6.4 \\
\hline 48 Mt 65 & $\begin{array}{l}\text { Southwest side of U. S. } \\
\text { road } 78 \text { (State road } 2 \text { ) } \\
\text { about } 27 / 8 \text { miles S } 87^{8} \\
\text { E of Ritgeville on north } \\
\text { side if Ashley River, } \\
\text { Dorchester County. }\end{array}$ & $\begin{array}{l}\text { Either Penholoway } \\
\text { terrace and forma- } \\
\text { tion or a dune along } \\
\text { the Pamlico terrace. }\end{array}$ & $40^{\prime}$ & Clayey material, & $\begin{array}{l}\text { Upper } 4 \text { feet of } \\
\text { 8-foot section }\end{array}$ & 25 & 5.3 \\
\hline $48 \mathrm{Mt} 66$ & " & $"$ & $40^{\prime}$ & Somewhat consolidated, & $\begin{array}{l}\text { Lower } 4 \text { feet of } \\
\text { 8-foot section }\end{array}$ & 25 & 7.8 \\
\hline $48 \mathrm{Mt} 67$ & $\begin{array}{l}\text { Northeast side of U. S. } \\
\text { road } 78 \text { (State road } 2 \text { ) } \\
\text { about } 3.3 \text { miles S } 72^{\mathrm{O}} \mathrm{E} \text { of } \\
\text { Ridgeville, and on south- } \\
\text { east side of Ashley River, } \\
\text { Dorchester County. }\end{array}$ & $\begin{array}{l}\text { Penholoway terrace } \\
\text { formation }\end{array}$ & $40^{\prime}$ & Clayej material. & 4-foot section & 25 & 4.3 \\
\hline $48 \mathrm{Nt} 68$ & $\begin{array}{l}\text { North side of U. S. road } \\
78 \text { (State road 2) ahout } \\
1.05 \text { miles } N 5^{\circ} \text { w of } \\
\text { Otranto, Bentey jounty. }\end{array}$ & $\begin{array}{l}\text { Talbot terrace and } \\
\text { fomation. }\end{array}$ & $30^{\prime}$ & $\begin{array}{l}\text { Semi-consolidated brown } \\
\text { sand. }\end{array}$ & 4-foot section & 25 & 13.9 \\
\hline 48 Mt 69 & $\begin{array}{l}\text { Northeast side of State } \\
\text { road } 31 \text {, about } 0.85 \text { mile } \\
\mathbb{N} 80^{\circ} \mathrm{W} \text { of Wassamassaw } \\
\text { Church and on west side } \\
\text { of Wassamassaw Swamp, } \\
\text { Berkeley County. }\end{array}$ & $\begin{array}{l}\text { Penholoway terrace } \\
\text { and formation }\end{array}$ & $50^{\prime}-60^{8}$ & $\begin{array}{l}\text { Mottled red, yellow, and } \\
\text { white, clayey sand stone. }\end{array}$ & 4-foot section & 25 & 12.0 \\
\hline
\end{tabular}


Table 1.--(Continued)

\begin{tabular}{|c|c|c|c|c|c|c|c|}
\hline Sample No. & Location & Formation & $\begin{array}{l}\text { itude } \\
\text { sea level) }\end{array}$ & Description & $\begin{array}{l}\text { Part of forma- } \\
\text { tion sampled }\end{array}$ & $\begin{array}{l}\text { Sample } \\
\text { weight } \\
\text { (pounds) } \\
\end{array}$ & $\begin{array}{l}\text { Heavy } \\
\text { mineral } \\
\text { weight } \\
\text { (grams) } \\
\end{array}$ \\
\hline 48 lit 70 & $\begin{array}{l}\text { Southeast side of U. S. } \\
\text { road } 15 \text {, about } 250 \text { yards } \\
\text { northeast of Lake Marione } \\
\text { (Santee River Dam) and } \\
100 \text { yards southeast of } \\
\text { the road, via rendon County. }\end{array}$ & $\begin{array}{l}\text { Either Wicomico } \\
\text { terrace or a dune } \\
\text { n the Penholoway } \\
\text { terrace and for- } \\
\text { nation. }\end{array}$ & $100^{\prime}$ & $\begin{array}{l}\text { Clayey, cream-colored } \\
\text { unconsolidated, very fine } \\
\text { sand, with disseminated } \\
\text { black specks of imertiti }\end{array}$ & $\begin{array}{l}\text { Upper } 4 \text { feet of } \\
\text { section }\end{array}$ & 25 & 26.8 \\
\hline $48 \mathrm{Mt} 71$ & $\begin{array}{l}\text { Road-metal pit on south- } \\
\text { east side of secondary } \\
\text { road, about } 1.62 \text { miles } N \\
35^{\circ} \text { W from bend in U. S. } \\
\text { road } 521 \text { west of Georgetown; } \\
\text { also } 100 \text { yards southwest of } \\
\text { junction of secondary road } \\
\text { with Johns on road, Georgetowr } \\
\text { County. }\end{array}$ & $\begin{array}{l}\text { Bar atop the } \\
\text { Pamlico terrace } \\
\text { and formation. } \\
\text { in }\end{array}$ & $25^{\prime}$ & Fine yellow sand. & 4-foot section & 25 & 20.9 \\
\hline $48 \mathrm{Mt} 72$ & $\begin{array}{l}\text { South bank of Black River, } \\
\text { about } 8 \text { miles } \mathrm{N} 13^{\circ} \mathrm{W} \text { from } \\
\text { Geor getown and } 700 \text { feet } \\
\text { west of the Pumping Station, } \\
\text { Geor getown County. }\end{array}$ & $\begin{array}{l}\text { Pamlico terrace } \\
\text { and formation. }\end{array}$ & $18^{\prime}$ & $\begin{array}{l}\text { Yellowish-brown sandy } \\
\text { clay. }\end{array}$ & 4-foot section & 25 & 14.0 \\
\hline $48 \mathrm{Mt} 73$ & $\begin{array}{l}\text { East side of Browns Ferry } \\
\text { road, about } 1.3 \text { miles } \\
\text { southeast of Browns Ferry, } \\
\text { Georgetown County. }\end{array}$ & $\begin{array}{l}\text { Shor eline of } \\
\text { Pamlico terrace } \\
\text { or pos sibly a } \\
\text { beach ridge on } \\
\text { the Talbot terrace } \\
\text { and formation. }\end{array}$ & $5^{2}-30^{1}$ & Yellow sand. & $6 \frac{1}{2}-$ foot section & 25 & 16.5 \\
\hline $48 \mathrm{Nit} 74$ & $\begin{array}{l}\text { Southwest side of Browns } \\
\text { Ferry road, about } 0.35 \\
\text { miles west-northwest of } \\
\text { Browns Ferry, Georgetown } \\
\text { County. }\end{array}$ & $\begin{array}{l}\text { Dune on shoreline of } \\
\text { Pamlico terrace and } \\
\text { formation. }\end{array}$ & $20^{\prime}$ & Fine yellow sand. & 4-foot section & 25 & 12.9 \\
\hline
\end{tabular}




\begin{tabular}{|c|c|c|c|c|c|c|c|}
\hline Sample No. & Location & Formation & $\begin{array}{c}\text { Altitude } \\
\text { (above sea level) }\end{array}$ & Description & $\begin{array}{l}\text { Part of forma- } \\
\text { tion sampled }\end{array}$ & $\begin{array}{l}\text { Sample } \\
\text { weight } \\
\text { (pounds) }\end{array}$ & $\begin{array}{l}\text { Heavy } \\
\text { mineral } \\
\text { weight } \\
\text { (grams) }\end{array}$ \\
\hline 48 Mt 75 & $\begin{array}{l}\text { Northeast side of Browns } \\
\text { Ferry road, about } 0.1 \text { mile } \\
\text { southeast of road junction } \\
\text { at Red Hill, Georgetown } \\
\text { County. }\end{array}$ & $\begin{array}{l}\text { Black Mingo } \\
\text { (lower Eocene) } \\
\text { formation. }\end{array}$ & $20^{\prime}-30^{\prime}$ & $\begin{array}{l}\text { Brownish-red to dark } \\
\text { red, well consolidated } \\
\text { sandstone and grit, that } \\
\text { disintegrates when panned. }\end{array}$ & 12-foot section & 25 & 31.3 \\
\hline $48 \mathrm{Mt} 76$ & $\begin{array}{l}\text { Northeast side of U. S. } \\
\text { road } 521 \text {, about } 1.63 \text { miles } \\
\text { northwest of Oak Grove and } \\
0.2 \text { miles northwest of } \\
\text { junction with Bethel Road, } \\
\text { Georgetown County. }\end{array}$ & $\begin{array}{l}\text { Talbot terrace } \\
\text { and formation. }\end{array}$ & $28^{1}$ & $\begin{array}{l}\text { Mottled red and orange } \\
\text { clayey sand; sandier at } \\
\text { base. }\end{array}$ & 4-foot section & 25 & 2.7 \\
\hline 48 Mt. 77 & $\begin{array}{l}\text { Northeast side of U. S. } \\
\text { road } 521 \text {, about } 0.57 \text { miles } \\
\text { N } 10^{\circ} \mathrm{E} \text {, of West Andrews, } \\
\text { Williamsburg County. }\end{array}$ & $\begin{array}{l}\text { Talbot terrace } \\
\text { and formation. }\end{array}$ & $35^{\prime}$ & $\begin{array}{l}\text { Upper } 2 \text { feet, leached } \\
\text { white sand; lower } 4 \text { feet, } \\
\text { yellow clay. }\end{array}$ & 6-foot section & 25 & 4.5 \\
\hline 48 Mt 78 & $\begin{array}{l}\text { Northeast side of U. S. } \\
\text { Road } 521 \text {, about } 4.8 \text { miles } \\
\text { wesi of Warsaw and } 200 \\
\text { yards east of Spring Gully } \\
\text { Church, Williamsburg County. }\end{array}$ & $\begin{array}{l}\text { Talbot terrace } \\
\text { and formation. }\end{array}$ & $40^{\prime}$ & $\begin{array}{l}\text { Upper } 3 \text { feet, dune } \\
\text { material; lower } 3 \text { feet, } \\
\text { yellowish-brown clayey } \\
\text { sandstone. }\end{array}$ & $\begin{array}{l}\text { Lower } 3 \text { feet } \\
\text { sampled }\end{array}$ & 25 & 12.4 \\
\hline 48 Mt 79 & $\begin{array}{l}\text { Northeast side of U. S. } \\
\text { road } 521 \text {, about } 6.85 \text { miles } \\
\text { IN } 77^{\circ} \mathrm{W} \text {. from Warsaw, and } \\
0.4 \text { miles southeast of } \\
\text { Spring Gulley, Williamsburg } \\
\text { County. }\end{array}$ & $\begin{array}{l}\text { Talbot terrace } \\
\text { and formation, } \\
\text { close to Talbot } \\
\text { shoreline. }\end{array}$ & $40^{\prime}$ & $\begin{array}{l}\text { Reddish-brown slightly } \\
\text { sandy clay. }\end{array}$ & 4-foot section & 25 & 3.8 \\
\hline
\end{tabular}


Table 1.--(Continued)

\begin{tabular}{|c|c|c|c|c|c|c|c|}
\hline Sample No. & Location & Formation & $\begin{array}{l}\text { Altitude } \\
\text { above sea level) }\end{array}$ & Description & $\begin{array}{l}\text { Part of forma- } \\
\text { tion sampled }\end{array}$ & $\begin{array}{l}\text { Sample } \\
\text { weight } \\
\text { (pounds) }\end{array}$ & $\begin{array}{l}\text { Heavy } \\
\text { mineral } \\
\text { weight } \\
\text { (grams) }\end{array}$ \\
\hline $48 \mathrm{Mt} 80$ & $\begin{array}{l}\text { Southwest side of State } \\
\text { road } 179 \text {, about } 1 \frac{1}{2} \text { miles } \\
\text { southeast of Honey Hill, } \\
\text { and just southeast of } \\
\text { Honey Hill Fire Tower, } \\
\text { Berkeley County. }\end{array}$ & $\begin{array}{l}\text { Shore line of } \\
\text { Pamlico terrace } \\
\text { and formation. }\end{array}$ & $25^{\prime}$ & $\begin{array}{l}\text { Light yellow unconsoli- } \\
\text { dated sand. }\end{array}$ & 3-foot section & 25 & 38.2 \\
\hline $48 \mathrm{Mt} 81$ & $\begin{array}{l}\text { West side of State road } \\
179 \text {, about } 0.6 \text { mile south- } \\
\text { east of Honey Hill, and } \\
0.83 \text { mile northwest of } \\
\text { Honey Hill Fire Tower, } \\
\text { Berkeley County. }\end{array}$ & $\begin{array}{l}\text { Dune on Talbot } \\
\text { terrace and } \\
\text { formation. }\end{array}$ & $40^{\prime}$ & $\begin{array}{l}\text { White sand, stained } \\
\text { yellow irregularly. }\end{array}$ & 6-foot section & 25 & 70.3 \\
\hline $48 \mathrm{Mt} 82$ & $\begin{array}{l}\text { East side of State road } \\
179 \text {, about } 1.7 \text { mile south- } \\
\text { east of Jamestown and about } \\
100 \text { feet northeast of junctic } \\
\text { with Pipkin road, Berkeley } \\
\text { County. }\end{array}$ & $\begin{array}{l}\text { Talbot terrace } \\
\text { and formation. } \\
\text { ion }\end{array}$ & $36^{\prime}$ & Brown sandy clay. & 3-foot section & 25 & 3.8 \\
\hline $48 \mathrm{Mt} 83$ & $\begin{array}{l}\text { Northwest side of State } \\
\text { road } 511 \text {, about } 1 \frac{1}{2} \text { miles } \\
\text { southwest of Jamestown, } \\
\text { and just southwest of } \\
\text { Tiger Corner road, Williams- } \\
\text { burg County. }\end{array}$ & $\begin{array}{l}\text { Talbot terrace } \\
\text { and formation. (?) }\end{array}$ & $40^{\prime}-45^{\prime}$ & $\begin{array}{l}\text { Consolidated red clay } \\
\text { containing mica but no } \\
\text { large grains of quartz. }\end{array}$ & 6-foot section & 25 & 3.0 \\
\hline $48 \mathrm{Mt} 84$ & $\begin{array}{l}\text { East side of State road } \\
511 \text {, about } 153 / 4 \text { miles } \\
\text { southwest of Jamestown, } \\
\text { Geor getown County. }\end{array}$ & $\begin{array}{l}\text { Talbot terrace } \\
\text { and formation. }\end{array}$ & $35^{\prime}$ & Red to orenge clay. & 10-foot section & 25 & 5.7 \\
\hline
\end{tabular}


Table 1.-(Continued)

\begin{tabular}{|c|c|c|c|c|c|c|c|}
\hline Sample No. & Location & $\begin{array}{l}\text { Altit } \\
\text { (above }\end{array}$ & $\begin{array}{l}\text { tude } \\
\text { sea level) }\end{array}$ & Description & $\begin{array}{l}\text { Part of forma- } \\
\text { tion sampled }\end{array}$ & $\begin{array}{l}\text { Sample } \\
\text { weight } \\
\text { (pounds) }\end{array}$ & $\begin{array}{l}\text { Heavy } \\
\text { mineral } \\
\text { weight } \\
\text { (grams) }\end{array}$ \\
\hline $48 \mathrm{Mt} 85$ & $\begin{array}{l}\text { North side of U. S. road } \\
\text { I7, about } 3 \text { miles east of } \\
\text { Awendaw, at Back Hall Fire } \\
\text { Tower, Charleston County. }\end{array}$ & $\begin{array}{l}\text { Offshore bar on } \\
\text { Pamlico terrace } \\
\text { and formation. }\end{array}$ & $20^{\prime}$ & $\begin{array}{l}\text { White to yellow un- } \\
\text { consolidated sand. }\end{array}$ & $\begin{array}{l}4 \text {-foot pit } \\
\text { section }\end{array}$ & 25 & 50.1 \\
\hline $48 \mathrm{Mt} 86$ & $\begin{array}{l}\text { West side of State road } \\
64 \text {, about } 7 \frac{1}{2} \text { miles north- } \\
\text { east of Summerville and } \frac{1}{4} \\
\text { mile north of Poplar } \\
\text { Branch, Berkeley County. }\end{array}$ & $\begin{array}{l}\text { Bar or dune on } \\
\text { Penholoway spit; } \\
\text { Penholoway terrace } \\
\text { and Oormation. }\end{array}$ & $70^{\prime}$ & $\begin{array}{l}3 \text { foot light yellow } \\
\text { unconsolidated sand, } \\
\text { underlain by yellow, } \\
\text { clayey sand. }\end{array}$ & 3 feet & 25 & 8.5 \\
\hline $48 \mathrm{Mt} 87$ & $\begin{array}{l}\text { North side of a side road } \\
\text { leading from U. S. road } 52 \\
\text { west to the Santeo Cooper } \\
\text { Hydroelectric Power Plant, } \\
\text { and } 0.7 \text { mile from U. S. } \\
\text { road } 52 \text {, Berkeley County. }\end{array}$ & Black Mingo formation. & $50^{\prime}$ & $\begin{array}{l}\text { Yellow-brown to orange } \\
\text { consolidated coarse } \\
\text { sandstone and grit } \\
\text { mottled with white spots } \\
\text { and stringers. }\end{array}$ & $\begin{array}{l}8 \text {-foot } \\
\text { section }\end{array}$ & 25 & 9.2 \\
\hline $48 \mathrm{Mt} 88$ & $\begin{array}{l}\text { Northeast side of U. S. } \\
\text { road } 52 \text {, about } 4 \text { miles } \\
\text { northwest of St. Stephens } \\
\text { at a point where a second- } \\
\text { ary road leads off to west; } \\
\text { about } 4 \text { miles southwest of } \\
\text { Santee River. }\end{array}$ & $\begin{array}{l}\text { Dune near Penholoway } \\
\text { shore line possibly } \\
\text { atop Wicomico terrace } \\
\text { and formation. }\end{array}$ & $77^{\prime}$ & $\begin{array}{l}6 \text { foot white unconsoli- } \\
\text { dated sand underlain by } \\
\text { yellow clayey sand. }\end{array}$ & $\begin{array}{l}6 \text {-foot } \\
\text { section }\end{array}$ & 25 & 76.7 \\
\hline
\end{tabular}


Table 2.-Heavy-mineral content of samples fmm Pleistocene terrace deposits and Eocene formations in southeastern South Garolina.

\begin{tabular}{|c|c|c|c|c|c|c|c|c|}
\hline Sample No. & Terjaces & $\begin{array}{c}\text { Total } \\
\text { heavy minerals } \\
\text { (percent) }\end{array}$ & $\begin{array}{l}\quad \text { Distri } \\
\text { Ilmenite } \\
\text { (percent) }\end{array}$ & $\begin{array}{l}\text { ibution of } \\
\text { teucoxene } \\
\text { (percent) }\end{array}$ & $\begin{array}{c}\text { total heavs } \\
\text { Rutile } \\
\text { (percent) }\end{array}$ & $\begin{array}{c}\mathrm{y} \text {-mineral } \\
\text { Zircon } \\
\text { (percent) }\end{array}$ & $\begin{array}{l}\text { content } \\
\text { Monazite } \\
\text { (percent) }\end{array}$ & $\begin{array}{l}\text { Others } \\
\text { Sercent }\end{array}$ \\
\hline 48 Mt 71 & Panlico & 0.18 & 68 & & 4 & 21 & 1 & 6 \\
\hline $48 \mathrm{Mt} 72$ & Panlico & .12 & 73 & 2 & 4 & 11 & 2 & 8 \\
\hline $48 \mathrm{kt} 73$ & Pamlico & .15 & 63 & & 5 & 28 & 1 & 3 \\
\hline $48 \mathrm{Mt} 74$ & Panlico & .14 & 74 & & 3 & 19 & 2 & 2 \\
\hline $48 \mathrm{Mt} 80$ & Pamlico & .34 & 58 & & 3 & 25 & $I$ & 13 \\
\hline $48 \mathrm{Mt} 85$ & Pamlico & .44 & 27 & $\operatorname{Tr}$ & $\operatorname{Tr}$ & 4 & $\operatorname{Tr}$ & 68 \\
\hline $48 \mathrm{Mt} 68$ & Talbot & .12 & 50 & 3 & 5 & 35 & 5 & 2 \\
\hline $48 \mathrm{Mt} 76$ & Talbot & .02 & 40 & 2 & 2 & 14 & $I$ & 41 \\
\hline 48 Nit 77 & Talbot &.$\alpha_{4}$ & 65 & 3 & 5 & 11 & 1 & 15 \\
\hline $48 \mathrm{Mt} 78$ & Talbot & .11 & 66 & 2 & 6 & 16 & 2 & 8 \\
\hline 48 lit 79 & Talbot & .03 & 54 & 3 & 3 & 24 & 3 & 13 \\
\hline $48 \mathrm{Mt} 81$ & Talbot & .62 & 62 & 3 & 6 & בI & & 18 \\
\hline 48 Mit 82 & Talbot & .03 & 50 & & 5 & 22 & & 23 \\
\hline $48 \mathrm{Mt} 83$ & Talbot & .03 & 71 & 1 & 2 & 14 & 2 & 10 \\
\hline $48 \mathrm{Nt} 84$ & Talbot & .05 & 67 & $\mathrm{Tr}$ & 3 & 16 & 3 & 11 \\
\hline $48 \mathrm{Mt} 63$ & Penholoway & .14 & 64 & 3 & 6 & 16 & 2 & 9 \\
\hline $48 \mathrm{Mt} 64$ & Penholoway & .06 & 45 & $\operatorname{Tr}$ & 8 & 24 & $\operatorname{Tr}$ & 23 \\
\hline 48 wt 65 & Penholoway & .05 & 61 & $\operatorname{Tr}$ & 3 & 21 & 5 & 10 \\
\hline $48 \mathrm{Mt} 66$ & Penholoway & .07 & 62 & $\operatorname{Tr}$ & 6 & 17 & 2 & 13 \\
\hline $48 \mathrm{Mt} 67$ & Penholoway &.$\alpha_{4}$ & 67 & & 4 & 18 & 1 & 10 \\
\hline $48 \mathrm{Mt} 69$ & Penholoway & .11 & 66 & $\mathrm{Tr}$ & 3 & 15 & 2 & 17 \\
\hline $48 \mathrm{Mt} 86$ & Penholoway & .07 & $6 I$ & & 5 & 30 & 3 & 1 \\
\hline $48 \mathrm{Mt} 88$ & Penholoway & .68 & 71 & & 3 & 17 & 2 & 13 \\
\hline $48 \mathrm{Mt} 59$ & Wicomico & .05 & 39 & & 9 & 27 & 4 & 31 \\
\hline
\end{tabular}


Table 2.--Heavy-mineral content of samples from Pleistocene terrace deposits and Eocerre formations in southeastern South Carolina (Continued)

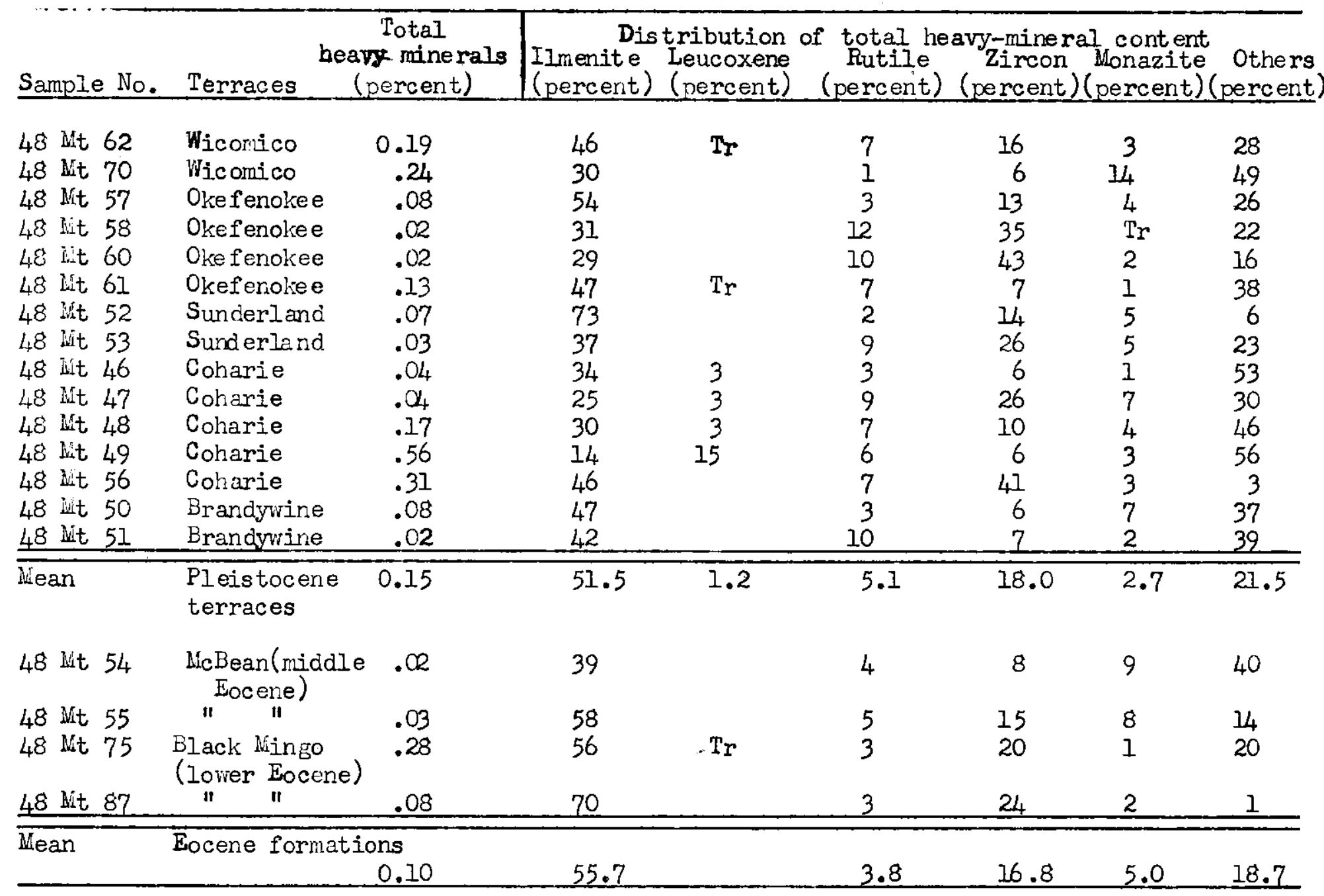


The amount of monazite in the heavy-mineral fraction of the Santee Velley terrace deposits, however, is greater thar elsewhero to the southwest along the Atlantic coast. Thus, the mineralogic analyses fram southeastern Georgia (table 4) show an average tenor in monasite of only 1.2 percent. This diminution in the content of monazite continues southward, as the tenor at the plent of the Titanium Alloy Manufacturing Co., southeast of Jecksonville, Fla., is 0.5 percent; and the tenor in monazite of the heavy minerals at the south end of Trail Ridge, Fla., Is from 0.1 to 0.05 percent. 


\section{Southeastern Georgia}

Twelve samples of the Plelstocene terrace deposits in the country between Brunswick and Trail Ridge were panned (fig. 2). One other sample from this area of the Miocene Hawthorn formation was also taken. The localities, altitudes, terrace assignments, lithology, and contents of heavy minerals for all these samples are given in tables 3 and 4.

The average content of heavy minerals in the terrace deposits of this area is more than twice that in the terrace deposits in and near the Santee Valley of South Carolina. Five of the samples taken from the east side of Trail Ridge have an average content of 0.6 percent of heavy minerals, about $1 / 7$ of the lowest commercial grade, and two of the five samples have a content of nearly 1.0 percent of heavy minerals, or approximately $1 / 4$ the lowest commercial grade.

These higher tenors of heavy minerals along the east side of Trafl Ridge may have some significance. It is true that no ores of commercial grade have been located, but if dependence had been placed entirely upon surficial sampling, the large deposit at the south end of Trall Ridge would not have been discovered, as the surficial tenor of heavy minerals is likewise about one percent. The commercial ore of this deposit, which has an average content of 3.9 percent of heavy minerals, lies from 10 to 40 feet below the surface. 


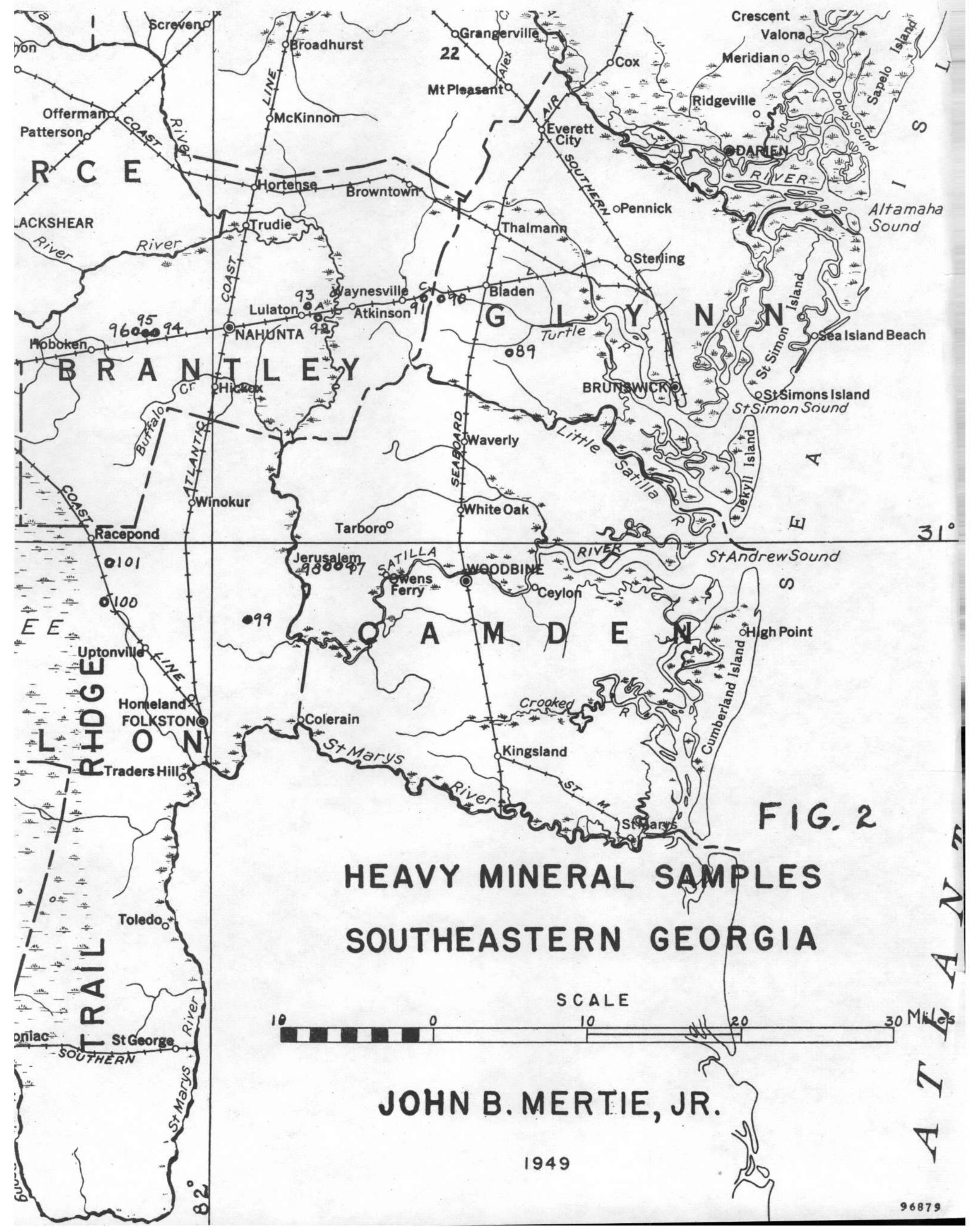


Table 3.-Localities and descriptions of samples from southeastem Georgia.

\begin{tabular}{|c|c|c|c|c|c|c|c|}
\hline Sample No. & Loc ation & $\begin{array}{r}\text { Alt } \\
\text { (above } \\
\end{array}$ & $\begin{array}{l}\text { titude } \\
\text { sea level) }\end{array}$ & Description & $\begin{array}{l}\text { Part of forma- } \\
\text { tion sampled }\end{array}$ & $\begin{array}{l}\text { Sample } \\
\text { weight } \\
\text { (pounds) }\end{array}$ & $\begin{array}{l}\text { Heavy } \\
\text { mineral } \\
\text { weight } \\
\text { (grams) }\end{array}$ \\
\hline $48 \mathrm{Mt} 89$ & $\begin{array}{l}\text { North side of U. S. road } \\
84 \text {, about } 2.3 \text { miles east } \\
\text { of College Creek, and } 2.7 \\
\text { miles east of junction } \\
\text { with State road } 99 \text {, Glynn } \\
\text { County. }\end{array}$ & $\begin{array}{l}\text { Probably bar on } \\
\text { Pamlico terrace } \\
\text { and formation }\end{array}$ & $?$ & Gray sand & $4 \frac{1}{2}$-foot section & 25 & 27.7 \\
\hline $48 \mathrm{kt} 90$ & $\begin{array}{l}\text { North side of U. S. road } \\
84 \text { about } 2.6 \text { miles east } \\
\text { of Waynesville, and } 2.8 \\
\text { miles west of Seaboard } \\
\text { RR crossing, Glynn County. }\end{array}$ & $\begin{array}{l}\text { Pamlico terrace } \\
\text { and formation near } \\
\text { Pamlico shor eline. }\end{array}$ & $2 I^{\prime}$ & $\begin{array}{l}\text { Upper half of section, } \\
\text { light gray, carbonaceous } \\
\text { sand; lower half, clayey } \\
\text { yellow sand. }\end{array}$ & $3 \frac{1}{2}$-foot section & 25 & 10.3 \\
\hline $48 \mathrm{Mt} 91$ & $\begin{array}{l}\text { North side of U. S. road } \\
84 \text {, about } 1 \frac{1}{2} \text { miles east } \\
\text { of Waynesville, Brantley } \\
\text { County. }\end{array}$ & $\begin{array}{l}\text { Outside bar on } \\
\text { Penholoway terrace } \\
\text { and formation }\end{array}$ & $60^{\prime}$ & $\begin{array}{l}\text { Light to dark yellow, non- } \\
\text { clayey, unconsolidated. } \\
\text { sand exposed in sand pit } \\
\text { at crest of hill. }\end{array}$ & 5-foot section & 25 & 14.2 \\
\hline $48 \mathrm{Nt} 92$ & $\begin{array}{l}\text { North side of U. S. road } \\
84 \text {, on crest of hill; about } \\
\text { l/2 mile west of Satlla } \\
\text { River, Brantley Count } y \text {. }\end{array}$ & $\begin{array}{l}\text { Bar or beach on } \\
\text { Penholoway terrace } \\
\text { and formation }\end{array}$ & $70^{\prime}$ & $\begin{array}{l}\text { White sand and exposed in } \\
\text { sand pit }\end{array}$ & 7-foot section & 25 & 8.9 \\
\hline $48 \mathrm{Mt} 93$ & $\begin{array}{l}\text { North side of U. S. road } \\
84 \text {, about } 0.7 \text { mile east } \\
\text { of Lulaton, and about } 5.6 \\
\text { miles west of railroad } \\
\text { crossing in Nahunta, } \\
\text { Brantley County. }\end{array}$ & $\begin{array}{l}\text { Hawthorn formation } \\
\text { (Miocene) ov erlain } \\
\text { by } 3 \text { feet of } \\
\text { Penholoway sand }\end{array}$ & $80^{\prime}$ & & $\begin{array}{l}7 \text {-foot section } \\
\text { of Hawthorn } \\
\text { formation }\end{array}$ & 25 & 8.7 \\
\hline $48 \mathrm{Nt} 94$ & $\begin{array}{l}\text { South side of U. S, road } \\
84 \text {, about } 1.2 \text { miles east } \\
\text { of Hoboken Five Tower, } \\
\text { Brantley County. }\end{array}$ & $\begin{array}{l}\text { Penholoway shoreline } \\
\text { on east side of } \\
\text { Trail Ridge. }\end{array}$ & $75^{\prime}$ & $\begin{array}{l}\text { Carbonaceous non-clayey } \\
\text { fairly coarse sand; } \\
\text { uniform grain size. }\end{array}$ & 5-foot section & 25 & 23.2 \\
\hline
\end{tabular}


Table 3.--(Continued)

\begin{tabular}{|c|c|c|c|c|c|c|c|}
\hline Sample No. & Location & $\begin{array}{l}\text { Altit } \\
\text { (above }\end{array}$ & $\begin{array}{l}\text { tude } \\
\text { sea level) }\end{array}$ & Description & $\begin{array}{l}\text { Part of forma- } \\
\text { tion sampled }\end{array}$ & $\begin{array}{l}\text { Sample } \\
\text { weight } \\
\text { (pounds) }\end{array}$ & $\begin{array}{l}\text { Heavy } \\
\text { mineral } \\
\text { weight } \\
\text { (grans) } \\
\end{array}$ \\
\hline $48 \mathrm{Mt} 95$ & $\begin{array}{l}\text { South side of U. S. road } \\
84 \text {, about } 0.5 \text { mile east of } \\
\text { Hoboken Five Tower, } \\
\text { Brantley County. }\end{array}$ & $\begin{array}{l}\text { Shore line of } \\
\text { Wicomico terrace and } \\
\text { formation on east side } \\
\text { of Trail Ridge }\end{array}$ & $100^{\prime}$ & $\begin{array}{l}\text { Coarse, carbonaceous } \\
\text { sand containing many } \\
\text { black minerals. }\end{array}$ & 4-foot section & 25 & 105.2 \\
\hline $48 \mathrm{Mt} 96$ & $\begin{array}{l}\text { Northside of U. S. road } \\
84 \text {, about } 200 \text { yards east } \\
\text { of Hoboken Five Tower, } \\
\text { Brantley County. }\end{array}$ & $\begin{array}{l}\text { Shore line of Oke-. } \\
\text { fenokee terrace and } \\
\text { formation on east } \\
\text { side of Trail Ridge. }\end{array}$ & $140^{\prime}$ & $\begin{array}{l}4 \text { feet of unconsoli- } \\
\text { dated gray, carbonaceous } \\
\text { sand lower } 4 \text { feet } \\
\text { is consolidated brown } \\
\text { sandstone that disintegrat } \\
\text { in water. }\end{array}$ & $\begin{array}{l}\text { 8-foot section } \\
\text { tes }\end{array}$ & 25 & 108.6 \\
\hline $48 \mathrm{Mt} 97$ & $\begin{array}{l}\text { South side of unpaved road, } \\
\text { about } 1 \frac{1}{4} \text { iles west-south- } \\
\text { west of verusalem, Camden } \\
\text { County. }\end{array}$ & $\begin{array}{l}\text { Above the level of the } \\
\text { Talbot terrace and } \\
\text { formation. }\end{array}$ & $45^{\prime}$ & $\begin{array}{l}\text { Hard packed white sand } \\
\text { at base, overlain by } \\
\text { carbonaceous sand, ov er- } \\
\text { lain by } 12 \text { inches of } \\
\text { windblown sand. }\end{array}$ & s-foot section & 25 & 28.0 \\
\hline $48 \mathrm{Mt} 98$ & $\begin{array}{l}\text { Northside of unpaved road, } \\
\text { about } 1 \text { I/ } 3 \text { miles west- } \\
\text { southwest of Jerusalem, } \\
\text { Cand en County. }\end{array}$ & $\begin{array}{l}\text { Penholoway terrace } \\
\text { and formation }\end{array}$ & $65^{\prime}$ & $\begin{array}{l}\text { Light yellow sand over- } \\
\text { lain by } 1 \text { foot of wind } \\
\text { blown sand. }\end{array}$ & 6-foot section & 25 & 22.8 \\
\hline 48 Mt 99 & $\begin{array}{l}\text { West side of Burnt Fork- } \\
\text { Folkston (unpaved) road } \\
\text { about } 2 / 5 \text { miles } S \text { F } \\
\text { from } \mathrm{W} \\
\text { Satella River) Charlton Count }\end{array}$ & $\begin{array}{l}\text { Penholoway terrace } \\
\text { and formation } \\
\text { ty. }\end{array}$ & $60^{\prime}$ & $\begin{array}{l}\text { White sand, resting on } \\
\text { Hawthorn formation. }\end{array}$ & 5-foot section & 25 & 35.4 \\
\hline $48 \mathrm{lit} 100$ & $\begin{array}{l}\text { West side of U. S. road } 1 \text {, } \\
32 / 5 \text { miles } \mathbb{N} \cdot 38^{\circ} \mathrm{W} \text { from } \\
\text { Uptonville, and on the } \\
\text { west side of the Atlantic } \\
\text { Coast Line, railroad cut: }\end{array}$ & $\begin{array}{l}\text { Wicomico terrace and } \\
\text { formation on east side } \\
\text { of Trail Ridge. }\end{array}$ & $100^{\prime}$ & Gray sand. & $\begin{array}{l}\text { Upper } 6 \text { feet of } \\
\text { cut }\end{array}$ & 25 & 62.0 \\
\hline
\end{tabular}


Table 3.--(Continued)

\begin{tabular}{|c|c|c|c|c|c|c|}
\hline Sample No. & Loc ation & $\begin{array}{c}\text { Altitude } \\
\text { (above sea level) } \\
\end{array}$ & Description & $\begin{array}{l}\text { Part of forma- } \\
\text { tion sampled }\end{array}$ & $\begin{array}{l}\text { Sample } \\
\text { weight } \\
\text { (pound s) }\end{array}$ & $\begin{array}{c}\text { Heavy } \\
\text { mineral } \\
\text { weight } \\
\text { (grans ) } \\
\end{array}$ \\
\hline $48 \mathrm{Nt} 101$ & $\begin{array}{l}\text { East side of U. S. road } \\
1,6 \text { I/4 miles } \mathrm{N}, 29^{\circ} \mathrm{W} \\
\text { from Uptonville at entrance } \\
\text { of a side road leading to } \\
\text { Sand HWl Churah, Charlton } \\
\text { County. }\end{array}$ & $\begin{array}{l}\text { Okefenoke } \\
\text { (Lower Sunderland } \\
\text { terrace and formation } \\
\text { along east side of } \\
\text { Trail Ridge). }\end{array}$ & $\begin{array}{l}\text { Yellow sand overlain } \\
\text { by thin bed of wind } \\
\text { blown sand. }\end{array}$ & 4 -foot section & 25 & 38.5 \\
\hline
\end{tabular}


Table 4.-Hieavy-mineral content of samples from Pleistocene terrace deposits of southeastern Georgia

\begin{tabular}{|c|c|c|c|c|c|c|c|c|}
\hline Sample No. & Terraces he & $\begin{array}{c}\text { Total } \\
\text { he avy minerals } \\
\text { (percent) }\end{array}$ & $\begin{array}{l}\text { Dis } \\
\text { Ilmenite } \\
\text { (percent) }\end{array}$ & $\begin{array}{l}\text { Stribution } \\
\text { Leucoxene } \\
\text { (percent) }\end{array}$ & $\begin{array}{c}\text { of total he } \\
\text { Rutile } \\
\text { (percent) }\end{array}$ & $\begin{array}{c}\text { avy-mine ra } \\
\text { Zircon } \\
\text { (percent) }\end{array}$ & $\begin{array}{l}\text { af content } \\
\text { Monazite } \\
\text { (percent) }\end{array}$ & $\left\{\begin{array}{l}\text { Others } \\
\text { (percent) }\end{array}\right.$ \\
\hline 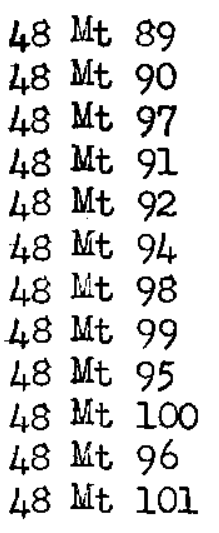 & $\begin{array}{l}\text { Panlico } \\
\text { Panlico } \\
\text { Talbot } \\
\text { Penholoway } \\
\text { Penholoway } \\
\text { Penholoway } \\
\text { Penholoway } \\
\text { Penholoway } \\
\text { Wicomico } \\
\text { Wicomico } \\
\text { Okefenokee } \\
\text { Oke fenokee }\end{array}$ & $\begin{array}{l}0.24 \\
.09 \\
.25 \\
.13 \\
.08 \\
.20 \\
.20 \\
.31 \\
.93 \\
.55 \\
.96 \\
.34\end{array}$ & $\begin{array}{l}47 \\
47 \\
50 \\
63 \\
49 \\
39 \\
47 \\
51 \\
31 \\
42 \\
43 \\
39\end{array}$ & $\begin{array}{l}2 \\
3 \\
\mathrm{Tr}_{\mathrm{r}} \\
\mathrm{Tr}\end{array}$ & $\begin{array}{l}7 \\
8 \\
5 \\
7 \\
6 \\
6 \\
9 \\
8 \\
4 \\
7 \\
4 \\
7\end{array}$ & $\begin{array}{l}38 \\
40 \\
38 \\
27 \\
38 \\
28 \\
27 \\
27 \\
24 \\
29 \\
27 \\
50\end{array}$ & $\begin{array}{l}6 \\
1 \\
1 \\
5 \\
\mathrm{Tr} \\
1 \\
\mathrm{Tr} \\
\mathrm{Tr} \\
\\
\mathrm{Tr}\end{array}$ & $\begin{array}{r}2 \\
5 \\
6 \\
2 \\
2 \\
27 \\
14 \\
17 \\
41 \\
22 \\
26 \\
4\end{array}$ \\
\hline Mean & $\begin{array}{c}\text { Pleis tocene } \\
\text { terraces }\end{array}$ & .37 & 45.7 & 0.4 & 6.5 & 32.7 & 1.2 & 13.5 \\
\hline 48 Mt 93 & $\begin{array}{l}\text { Hawthorn } \\
\text { (Mflocere.) }\end{array}$ & 0.08 & 67 & & 5 & 18 & 8 & 2 \\
\hline
\end{tabular}




\section{CONCLUSIONS}

If economic concentrations of heavy minerals exist in the Pleistocene terraces of South Carolina and Georgia, they most likely occur in the Wicomico, Okefenokee, and Sunderland terraces and formations, and to the east or southeast of Trail Ridge. Analyses of samples show that sands containing relatively large amounts of heavy minerals are percentagewlse low in monazite, and that sands containing small amounts of heavy minerals are percentagewise high in monazite.

It does not seem likely that further surface sampling will be of value in searching for such deposits. The formations in which heavy minerals occur are not indicated by the topography, and the sites of particular Pleistocene shore lines are no index of the occurrence of ore sands. Spots of black sand along the present beaches are perhaps the least significant indices. Physiographic and general geologic studies are therefore poor exploratory tools, though geologic and mineralogic work are needed in genetic studies of the ore sand.

Exploration by drilling and trenching is the only means of locating new deposits. Auger drilling and trenching might suffice for shallow prospecting but, owing to the water tables' proximity to the surface, deeper exploration would be done best by drilling with casing. Exploration by drilling requires equipment, skilled personnel, and laboratory facilities for the study of samples; therefore, any program of drilling would have to provide for these items. 

